\title{
CENTRO DE GRAVIDADE PARA LOCALIZAR CENTRO DE COLETA PARA A ECONOMIA CIRCULAR DO ÓLEO DE COZINHA EM VITÓRIA, ES
}

\author{
GRAVITY CENTER TO LOCATE A COLLECTION CENTER FOR THE CIRCULAR ECONOMY OF \\ COOKING OIL IN VITÓRIA, ES
}

\author{
Bárbara Nascimento Bittencourt ${ }^{1} \&$ Marcos Wagner Jesus Servare Junior ${ }^{2 *}$ \\ 12 Centro Universitário Salesiano - Unisales \\ ${ }^{1}$ nb_barbara@hotmail.com ${ }^{2 *}$ marcos.servare@salesiano.br
}

\section{ARTIGO INFO.}

\section{Recebido em: 16.11.2021}

Aprovado em: 10.12.2021

Disponibilizado em: 14.12.2021

Palavras-chave:

Gestão de Resíduos; Centro de Gravidade; Óleo de Cozinha; Centro de Coleta.

KEYWORDS: Waste Management; Gravity Center; Cooking oil; Collection Center.

*Autor Correspondente: Servare Junior, M. W. J.

\section{RESUMO}

O aumento da geração de resíduos faz com que seja necessária a implantação de uma série de ações para evitar os problemas ambientais, sendo essa uma responsabilidade compartilhada de toda a sociedade. Logo, o presente artigo tem como objetivo indicar através do método do centro de gravidade a melhor localização de um ponto de coleta de óleo de cozinha no município de Vitória, evidenciando a aplicabilidade do método escolhido para definir a localização. A partir da metodologia escolhida foi realizada uma revisão de literatura na qual se divide em dois temas, gestão de resíduos e aplicação do método do centro de gravidade, contribuindo para a contextualização do tema da pesquisa destacando a relevância do mesmo. Entretanto, considerando o método escolhido foram listados os 80 bairros do município, bem como as coordenadas geográficas dos mesmos. Além disso, foram utilizadas as demandas de consumo em cada bairro nos cenários de 5 e 15 anos. No entanto, a partir da técnica escolhida foi possível observar que a localização proposta está entre os bairros com o maior consumo de óleo de cozinha.

\begin{abstract}
The increase in waste generation makes it necessary to implement a series of actions to avoid environmental problems, which is a shared responsibility of the entire society. Therefore, this article aims to indicate, through the center of gravity method, the best location of a cooking oil collection point in the city of Vitoria, showing the applicability of the method chosen to define the location. From the chosen methodology, a literature review was carried out, which is divided into two themes, waste management and application of the center of gravity method, contributing to the contextualization of the research theme, highlighting its relevance. However, considering the chosen method, the 80 districts of the city were listed, as well as their geographic coordinates. In addition, consumption demands in each neighborhood in the 5- and 15-year scenarios were used. However, from the chosen technique, it was possible to observe that the proposed location is among the neighborhoods with the highest consumption of cooking.
\end{abstract}



circular do óleo de cozinha em Vitória, ES. Brazilian Journal of Production Engineering, 7(5), 194-206.

\section{INTRODUÇÃO}

O aumento da geração de resíduos requer uma série de ações, desde a manipulação, tratamento e descarte adequado com o intuito de evitar problemas ambientais. Na maioria dos casos, a motivação para tratamento e descarte adequados dos resíduos surge na forma de imposição legal, devido ao fato de que é o aumento destas exigências que faz com que a sociedade se adeque e busque novas estratégias de resolução dos problemas ambientais (Almeida, 2014).

De acordo com a Política Nacional de Resíduos Sólidos (PNRS), a responsabilidade pelo ciclo de vida dos produtos é compartilhada, sendo ela um dever desde os fabricantes, passando pelos consumidores, chegando até os titulares dos serviços públicos de limpeza urbana e de manejo dos resíduos sólidos, com o intuito de minimizar o volume de resíduos sólidos e rejeitos gerados (Soares, Biagio, Gonçalves, \& Servare Junior, 2020).

O Projeto de Lei do Senado 75/2017, de autoria do senador José Medeiros que visa alterar a Lei $\mathrm{n}^{\mathrm{o}}$ 12.305/2010 da PNRS para a inclusão do óleo de cozinha na lista de produtos do sistema de logística reversa. Esta inclusão justifica-se pelo fato de que o óleo de cozinha quando descartado de maneira incorreta, por exemplo, quando em contato com a água chega a contaminar em torno de até 10 mil litros de água (Brasil, 2017).

Logo, todo este resíduo deve ser encaminhado a um ponto de coleta onde será reutilizado, porém em muitos casos ocorre a falta de informação ou há uma dificuldade de acesso a esses pontos, surgindo então a necessidade de um centro de coleta desse óleo com uma localização que possa atender melhor a população.

Neste contexto, o presente artigo tem como objetivo utilizar uma das técnicas de localização para propor a melhor localização para a implantação de um centro de coleta de óleo de cozinha residual no município de Vitória, Espírito Santo, utilizando o método do Centro de Gravidade. Considerando a demanda de consumo dos bairros do município e suas coordenadas geográficas.

Portanto, neste artigo a primeira seção permitirá a compreensão do tema através da introdução. A segunda seção apresenta uma revisão literária que é dividida em dois temas, gestão de resíduos e a aplicação do método do centro de gravidade, identificando a importância desse tipo de gestão e destacando a aplicabilidade do método escolhido para definir a localização.

Na sequência a importância da economia circular do óleo de cozinha na terceira seção, em seguida na quarta seção implantação do método do centro de gravidade, incluindo os dados coletados para a pesquisa, por fim, a quinta seção apresenta os resultados e as considerações finais obtidas ao longo do desenvolvimento do trabalho.

\section{REVISÃO DE LITERATURA}

\subsection{GESTÃO DE RESÍDUOS SÓLIDOS}

Segundo Nascimento, Sobral, Andrade, \& Ometto (2015) a geração dos resíduos sólidos urbanos (RSU) no Brasil cresce a cada dia mais ao longo do tempo, com alterações na qualidade do resíduo, fazendo com o que as discussões a respeito do gerenciamento e da importância da destinação destes resíduos sejam levantadas. 
Citação (APA): Bittencourt, B. N. \& Servare Junior, M. W. J. (2021). Centro de gravidade para localizar centro de coleta para a economia circular do óleo de cozinha em Vitória, ES. Brazilian Journal of Production Engineering, 7(5), 194-206.

Nascimento et al. (2015) utilizou uma análise bibliométrica abordando algumas reflexões sobre o PNRS, tendo em vista os aspectos esperados para acontecer no setor público após a implementação da Política Nacional de Resíduos Sólidos. Por fim, a pesquisa conclui que houve uma melhora relacionada ao gerenciamento dos RSU no Brasil, porém não foi o suficiente para cumprir as propostas feitas pela PNRS, sendo necessário o estabelecimento de uma gestão integrada de RSU mais eficaz e sustentável ao meio ambiente e a sociedade.

Fonseca et al. (2015) realizou uma análise bibliométrica dos artigos publicados entre os anos de 2010 a 2014, com objetivo de avaliar a evolução de estudos de Logística Reversa (LR) no contexto brasileiro. Esta análise também tem como base a Política Nacional de Resíduos Sólidos (PNRS). A partir dos resultados da pesquisa, identificou-se uma tendência de desenvolvimento de estudos relacionados, porém, foram identificadas algumas lacunas de conhecimento que viabilizam o desenvolvimento de novas pesquisas em Logística Reversa associadas ao contexto da implementação da PNRS.

Dentre os quatros artigos selecionados que abordam o tema de gestão de resíduos sólidos, dois deles trazem uma análise da aplicação desta gestão em dois municípios brasileiros, através de estudos de casos. Monteiro, Karpinski, Kuhl, \& Morozini (2016), propuseram um estudo com o objetivo de identificar e analisar aspectos da sustentabilidade na gestão municipal de resíduos sólidos em um município do Centro Oeste do Paraná, é caracterizado como um estudo de caso de caráter descritivo e com escopo quantitativo.

A pesquisa foi aplicada no município de Candói, localizado na região Centro Oeste do Paraná. A mesma é classificada como descritiva, visto que foi utilizada para relatar sobre os fenômenos que se relacionam aos aspectos da sustentabilidade na gestão municipal de resíduos sólidos. Foram aplicadas as técnicas estatísticas com base na estatística descritiva, através do pacote estatístico Statistical Package for the Social Sciences (SPSS) (Monteiro et al., 2016).

Através do método aplicado dentre as informações obtidas, os dados coletados mostraram que $61 \%$ dos pesquisados consideram a sustentabilidade muito importante para suas vidas, apenas $2 \%$ se mostrou indiferente em relação à sustentabilidade. Por fim, concluiu-se que supostamente a gestão municipal de resíduos sólidos do município, ainda não está perfeitamente alinhado com as demais dimensões ou que ainda não seja do conhecimento de toda população (Monteiro et al., 2016).

Já o estudo proposto por Abreu, Silva, Santos M., \& Santos L. (2020) faz uma análise sobre a gestão de resíduos sólidos domésticos na cidade de Macaé teve como objetivo identificar e analisar aspectos da sustentabilidade na gestão municipal de resíduos, identificando o perfil, nível de conhecimento e a aceitabilidade das medidas de gestão dos resíduos domésticos. Para isso, realizou-se um estudo de caso de caráter descritivo e com escopo quantitativo, foram utilizados questionário e formulário para coletar os dados

Utilizou-se a ferramenta do Google Forms para conhecer as características do perfil dos moradores da cidade, procuraram também dados oficiais da Prefeitura de Macaé divulgados pelo site, para que todos os questionamentos fossem respondidos. Com base nos resultados obtidos foi possível concluir que houve um grande interesse da população em colaborar com a 
Citação (APA): Bittencourt, B. N. \& Servare Junior, M. W. J. (2021). Centro de gravidade para localizar centro de coleta para a economia circular do óleo de cozinha em Vitória, ES. Brazilian Journal of Production Engineering, 7(5), 194-206.

gestão dos resíduos, sustentando a necessidade de implementação de projetos ou programas municipais que incentivem a coleta seletiva (Abreu et al., 2020).

De acordo na semelhança do contex to dos artigos analisados, foi elaborado o Quadro 1 com os principais pontos abordados dos mesmos, com o intuito de facilitar a compreensão de suas características, bem como promover a organização da análise desta seção apresentada.

Quadro 1. Artigos selecionados com o tema gestão de resíduos

\begin{tabular}{|c|c|c|c|}
\hline Artigo & Objetivo & Metodologia & Principais resultados \\
\hline $\begin{array}{c}\text { Nascimento } \\
\text { et al (2015) }\end{array}$ & $\begin{array}{c}\text { Abordar aspectos sobre os } \\
\text { resíduos sólidos no Brasil }\end{array}$ & $\begin{array}{c}\text { Análise bibliométrica } \\
\text { abordando algumas } \\
\text { reflexões sobre o } \\
\text { PNRS }\end{array}$ & $\begin{array}{c}\text { As melhorias ocorridas através } \\
\text { do gerenciamento dos RSU não } \\
\text { foram suficientes para que as } \\
\text { mudanças propostas pela PNRS } \\
\text { fossem cumpridas. }\end{array}$ \\
\hline $\begin{array}{c}\text { Fonseca et } \\
\text { al. } \text { (2015) }\end{array}$ & $\begin{array}{c}\text { Avaliar a evolução de estudos de } \\
\text { Logística Reversa no contexto } \\
\text { brasileiro }\end{array}$ & $\begin{array}{c}\text { Análise bibliométrica } \\
\text { de artigos publicados } \\
\text { entre os anos de 2010 } \\
\text { e 2014. }\end{array}$ & $\begin{array}{c}\text { Identificou-se o crescimento no } \\
\text { número de publicações sobre o } \\
\text { tema analisado. }\end{array}$ \\
\hline $\begin{array}{c}\text { al. } \text { (2016) } \\
\text { Identificar e analisar aspectos da } \\
\text { sustentabilidade na gestão } \\
\text { municipal de resíduos sólidos em } \\
\text { um município do Centro Oeste do } \\
\text { Paraná. }\end{array}$ & $\begin{array}{c}\text { Um estudo de caso de } \\
\text { caráter descritivo e } \\
\text { com escopo } \\
\text { quantitativo. }\end{array}$ & $\begin{array}{c}\text { Supostamente a gestão } \\
\text { municipal de resíduos sólidos } \\
\text { do município, ainda não está } \\
\text { perfeitamente alinhado com as } \\
\text { demais dimensões ou que ainda } \\
\text { não seja do conhecimento de } \\
\text { toda população. }\end{array}$ \\
\hline $\begin{array}{c}\text { Abreu et al. } \\
\text { (2020) }\end{array}$ & $\begin{array}{c}\text { Identificar as ações do município } \\
\text { de Macaé frente à gestão de } \\
\text { resíduos sólidos domésticos e o } \\
\text { destino dado a eles. }\end{array}$ & $\begin{array}{c}\text { Estudo exploratório e } \\
\text { descritivo }\end{array}$ & $\begin{array}{c}\text { As medidas acerca da gestão } \\
\text { ambiental de resíduos sólidos } \\
\text { precisam ser ampliadas e } \\
\text { difundidas no município }\end{array}$ \\
\hline
\end{tabular}

A partir da análise destes artigos foi possível verificar que segundo os autores, a geração de resíduos sólidos no Brasil cresce cada dia mais ao longo do tempo, e também é visível uma mudança na qualidade destes resíduos, fazendo com o que as discussões a respeito do gerenciamento e da importância da destinação dos mesmos sejam levantadas. Entretanto, quanto à gestão analisada nos municípios, é possível observar que em muitos casos ocorre a falta de informação e que há necessidade de implementação de projetos ou programas municipais que incentivem e informem a população sobre o gerenciamento dos resíduos.

\subsection{MÉTODO DO CENTRO DE GRAVIDADE}

O método do centro de gravidade é utilizado para definir uma localização evidenciando a minimização de custo de transporte. Além de procurar avaliar o local levando em consideração o menor custo, o mesmo considera os mercados consumidores e o fornecimento de matériasprimas, sendo o método mais utilizado na atualidade (Soares et al., 2020).

O estudo proposto por Soares et al. (2020) tem como objetivo identificar a melhor localização para a instalação do Centro de Triagem da logística reversa de aparas de papel gerados pela escola do bairro Vila Mariana em São Paulo. Este estudo também utiliza a PNRS como referência.

Entretanto, foi utilizado o método do centro de gravidade, onde foram selecionadas as escolas com a localidade no cadastro na secretaria da educação. Foram encontradas variáveis de decisão 
Citação (APA): Bittencourt, B. N. \& Servare Junior, M. W. J. (2021). Centro de gravidade para localizar centro de coleta para a economia circular do óleo de cozinha em Vitória, ES. Brazilian Journal of Production Engineering, 7(5), 194-206.

como, a quantidade de alunos por escola, quantidade de resíduo gerado e as distâncias, que influenciaram no desenvolvimento do método. Para facilitar a aplicação das fórmulas do método foi utilizada em longitude (X) e latitude (Y). Sendo assim, após a realização dos cálculos foi constatada a melhor localização, observando um custo mensal de transporte superior ao ponto ótimo (Soares et al., 2020).

Já no estudo proposto por Maia, Rodrigues, \& Moraes (2020) o método apresentado é aplicado com o intuito de apresentar a melhor localização para a instalação futura de um centro de distribuição (CD) de uma fábrica de argamassa e rejunte. O estudo foi desenvolvido através das seguintes etapas: a) Identificação dos principais clientes; b) Seleção dos três clientes responsáveis pela maior movimentação de volumes; c) Localização das coordenadas dos mesmos através do Google Maps; d) Cálculo do centro de gravidade.

A partir dos resultados obtidos após a implantação do método, foi observado que a localidade proposta é vantajosa por não ocasionar tanto impacto para os colaboradores, mantendo a proximidade também com seus principais clientes, entre outros fatores vantajosos. O método Centro de Gravidade considera as localizações existentes das fontes de insumo, mercado consumidor e os gastos envolvidos no transporte do volume desses bens e serviços, para que as coordenadas encontradas após a aplicação do modelo apontem um ponto, o centro de gravidade, em que caso essa organização seja instalada nesse local, os dispêndios com transporte serão minimizados (Maia et al., 2020).

Após a conclusão dos estudos nos dois casos apresentados, de acordo com Maia (2020) e Soares (2020), devido à eficácia do método do centro de gravidade, é possível calcular o melhor ponto de localização. Entretanto, a sua análise traz um direcionamento quantitativo para a decisão de localização, porém falha ao não analisar de forma mais criteriosa os aspectos qualitativos que também possuem grande impacto na decisão da localização.

\section{IMPORTÂNCIA DA ECONOMIA CIRCULAR DO ÓLEO DE COZINHA}

De acordo com Leitão (2015) a Economia Circular é um modelo que permite a mudança de perspectiva, passando da perspectiva linear (extrair, produzir, consumir e descartar) para uma visão circular que se preocupa com o que ocorre após o consumo e descarte. Inclui-se num quadro de desenvolvimento sustentável baseado no princípio de fechar o ciclo de vida dos produtos, permitindo a redução no consumo de matérias-primas.

Segundo a Política Nacional de Resíduos Sólidos (PRNS) o óleo de cozinha é um dos produtos bastante utilizado como base da cozinha brasileira no preparo dos alimentos e está presente em várias receitas do cotidiano. Em alguns casos devido à falta de informação, o resíduo deste insumo é descartado de maneira incorreta em ralos de pias ou diretamente no solo, ocasionando graves impactos ao meio ambiente, visto que um litro de óleo pode contaminar cerca de vinte litros de água (Servare Junior \& Cardoso, 2020).

Sendo assim, de acordo com Pitta Junior et al., (2009) este resíduo requer uma gestão adequada que diminua os impactos causados ao meio ambiente e a problemas no sistema de tratamento de água e esgoto, uma das opções corretas para esta gestão é a destinação deste óleo residual para algum ponto de coleta, no qual após a coleta o mesmo possa se tornar matéria prima para 
Citação (APA): Bittencourt, B. N. \& Servare Junior, M. W. J. (2021). Centro de gravidade para localizar centro de coleta para a economia circular do óleo de cozinha em Vitória, ES. Brazilian Journal of Production Engineering, 7(5), 194-206.

outros produtos, como a produção de sabão, óleos para engrenagens, biodiesel, detergentes, entre outros.

\section{LOCALIZAÇÃO A PARTIR DO MÉTODO DO CENTRO DE GRAVIDADE}

Segundo Slack et al. (2015) no âmbito empresarial, para escolher a localização é necessário ter equilíbrio em três objetivos principais, sendo eles, os custos variáveis, o tipo de serviço que é prestado e o potencial de faturamento da operação. Os custos variáveis estão ligados ao custo de mão de obra, custo de transporte, energia, entre outros. Já o objetivo referente ao serviço que é prestado, dependendo de fatores como: O tipo de serviço, a divulgação do mesmo, que pode ser influenciada pelo lugar onde ele ocorre, e por último, o potencial de faturamento da operação é influenciado diretamente pela demanda, custos de oferta, e outros fatores que estão diretamente ligados ao seu posicionamento.

De acordo com Oliveira, Neto, kwaitkowski da Silva e Santoro (2015), o método do centro de gravidade é utilizado para resolver problemas de localização de instalação única. Com este método pode ser avaliado a demanda existente, e o volume de bens ou serviços, visando a minimização de custos de transporte. Para calcular as distâncias, em geral é colocado um eixo cartesiano sobre o mapa da região escolhida e é determinada a posição dos centros de demanda em relação a uma origem aleatória.

Martins e Laugeni (2015) afirmam que o método procura avaliar o local de menor custo para a instalação, considerando os mercados consumidores e o fornecimento dos insumos necessários . São utilizadas duas expressões (1) e (2), uma para cada coordenada da localização, que são utilizadas para definir as coordenadas no cálculo do centro de gravidade, sendo chamadas também de coordenadas do centro de gravidade.

$$
\begin{aligned}
& y=\frac{\sum \quad \text { ViCiyi }}{\sum \quad \text { Viyi }} \\
& x=\frac{\sum \quad \text { ViCixi }}{\sum \quad \text { Vixi }}
\end{aligned}
$$

Sendo:

$V i=$ Volume em transportado para o local i;

$C i=$ Custo $(\mathrm{R} \$)$ por unidade de volume transportado para o local $\mathrm{i}$;

$x i=$ Coordenada na direção $\mathrm{x}$ do local $\mathrm{i}$;

$y i=$ Coordenada na direção y do local i.

\subsection{COLETA DE DADOS}

Nesta seção serão apresentados os valores e modo de obtenção dos dados empregados como parâmetro para a aplicação da técnica do Centro de Gravidade, com o intuito de propor a melhor localização para um centro de coleta de óleo de cozinha no município de Vitória. O desenvolvimento do estudo se deu a partir da listagem dos bairros do município analisado, resultando em 80 bairros no total. Em seguida foram realizadas pesquisas no site Google Maps 
Citação (APA): Bittencourt, B. N. \& Servare Junior, M. W. J. (2021). Centro de gravidade para localizar centro de coleta para a economia circular do óleo de cozinha em Vitória, ES. Brazilian Journal of Production Engineering, 7(5), 194-206.

(2021) com o objetivo de obter as coordenadas geográficas x e y de cada bairro listado. Veja a relação dos bairros analisados no Quadro 2.

Quadro 2. Lista dos bairros do município de Vitória.

\begin{tabular}{|c|c|c|c|}
\hline Aeroporto & Enseada do Suá & Joana D'Arc & Santa Cecília \\
\hline Andorinhas & Estrelinha & Jucutuquara & Santa Clara \\
\hline Antonio Honório & Fonte Grande & Maria Ortiz & Santa Helena \\
\hline Ariovaldo Falavessa & Forte São João & Mário Cypreste & Santa Lúcia \\
\hline Barro Vermelho & Fradinhos & Maruípe & Santa Luíza \\
\hline Bela Vista & Goiabeiras & Mata da Praia & Santa Martha \\
\hline Bento Ferreira & Grande Vitória & Monte Belo & Santa Tereza \\
\hline Boa Vista & Gurigica & Morada de Camburi & Santo André \\
\hline Bonfim & Horto & Nazareth & Santo Antonio \\
\hline Caratoíra & Ilha das Caieiras & Nova Palestina & Santos Dumont \\
\hline Centro & Ilha de Santa Maria & Parque Industrial & Santos Reis \\
\hline Comdusa & Ilha do Boi & Parque Moscoso & São Benedito \\
\hline Conquista & Ilha do Frade & Piedade & São Cristóvão \\
\hline Consolação & Ilha do Príncipe & Pontal de Camburi & São José \\
\hline Cruzamento & Inhanguetá & Praia do Canto & São Pedro \\
\hline Da Penha & Itararé & Praia do Suá & Segurança do Lar \\
\hline De Lourdes & Jabour & Redenção & Solon Borges \\
\hline Do Cabral & Jardim Camburi & República & Tabuazeiro \\
\hline Do Moscoso & Jardim da Penha & Resistência & Universitário \\
\hline Do Quadro & Jesus de Nazareth & Romão & Vila Rubim \\
\hline
\end{tabular}

Em sequência, foram utilizadas as demandas de litros de óleo de cozinha consumidos em cada bairro listado. Estas demandas foram obtidas através dos resultados de um modelo matemático proposto por Servare Junior \& Cardoso (2020), no qual foi analisado o problema de postergação de tempo e proposto uma modelagem matemática para utilização da postergação de tempo no projeto da cadeia de suprimentos reversa.

As demandas de consumo se encontram no cenário de 5 e 15 anos, de acordo com os autores, esses valores foram escolhidos por englobar o planejamento em médio e longo prazo e propor soluções para um período de tempo que incentive a instalação proposta. Com isso, o Figura $1 \mathrm{e}$ 2 apresenta a demanda de consumo dos bairros no cenário de 5 e 15 anos, respectivamente. (Servare Junior \& Cardoso, 2020) 
Citação (APA): Bittencourt, B. N. \& Servare Junior, M. W. J. (2021). Centro de gravidade para localizar centro de coleta para a economia circular do óleo de cozinha em Vitória, ES. Brazilian Journal of Production Engineering, 7(5), 194-206.

Figura 1. Demanda dos bairros do município de Vitória em 5 anos.

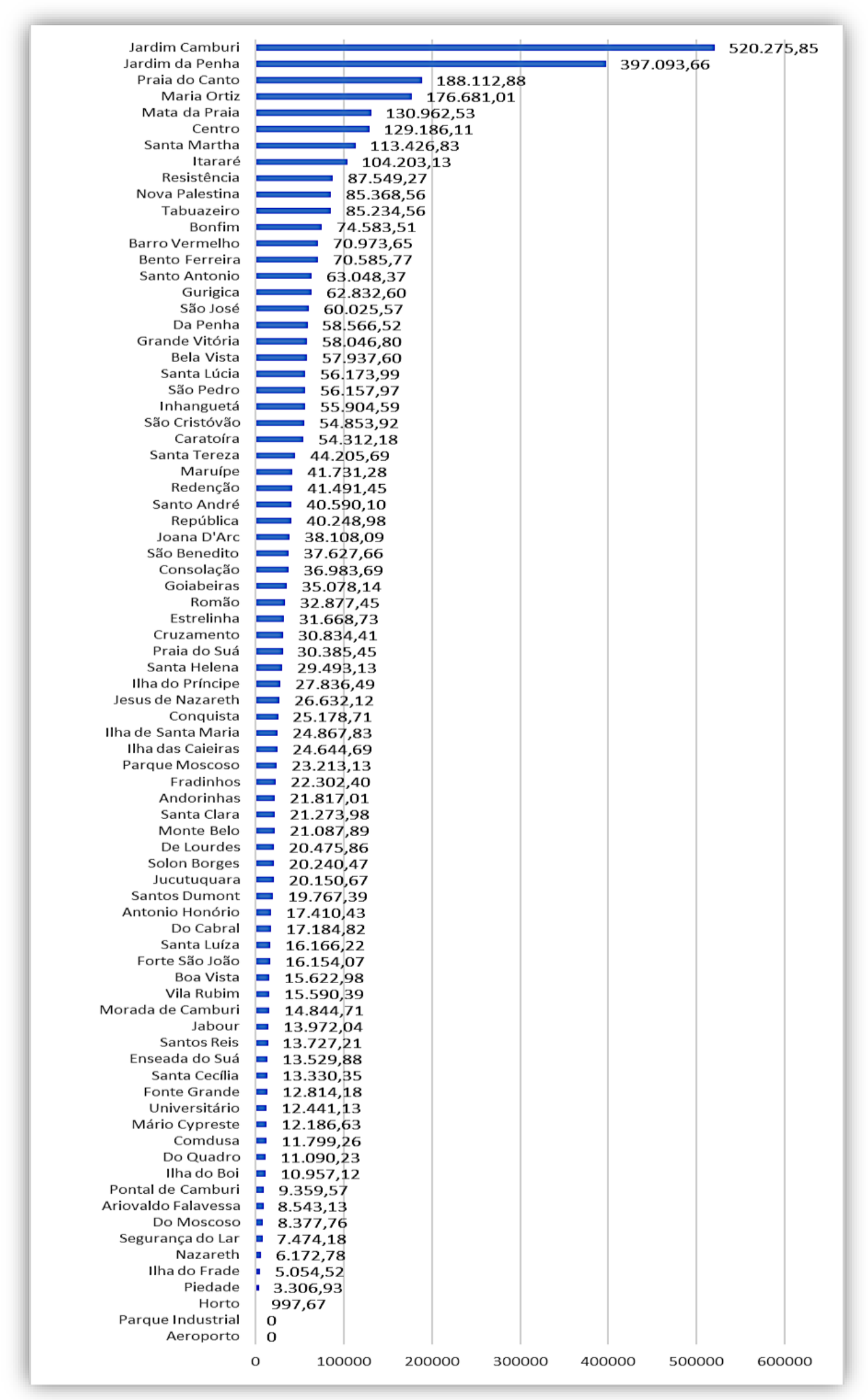


Citação (APA): Bittencourt, B. N. \& Servare Junior, M. W. J. (2021). Centro de gravidade para localizar centro de coleta para a economia circular do óleo de cozinha em Vitória, ES. Brazilian Journal of Production Engineering, 7(5), 194-206.

Figura 2. Demanda dos bairros do município de Vitória em 15 anos.

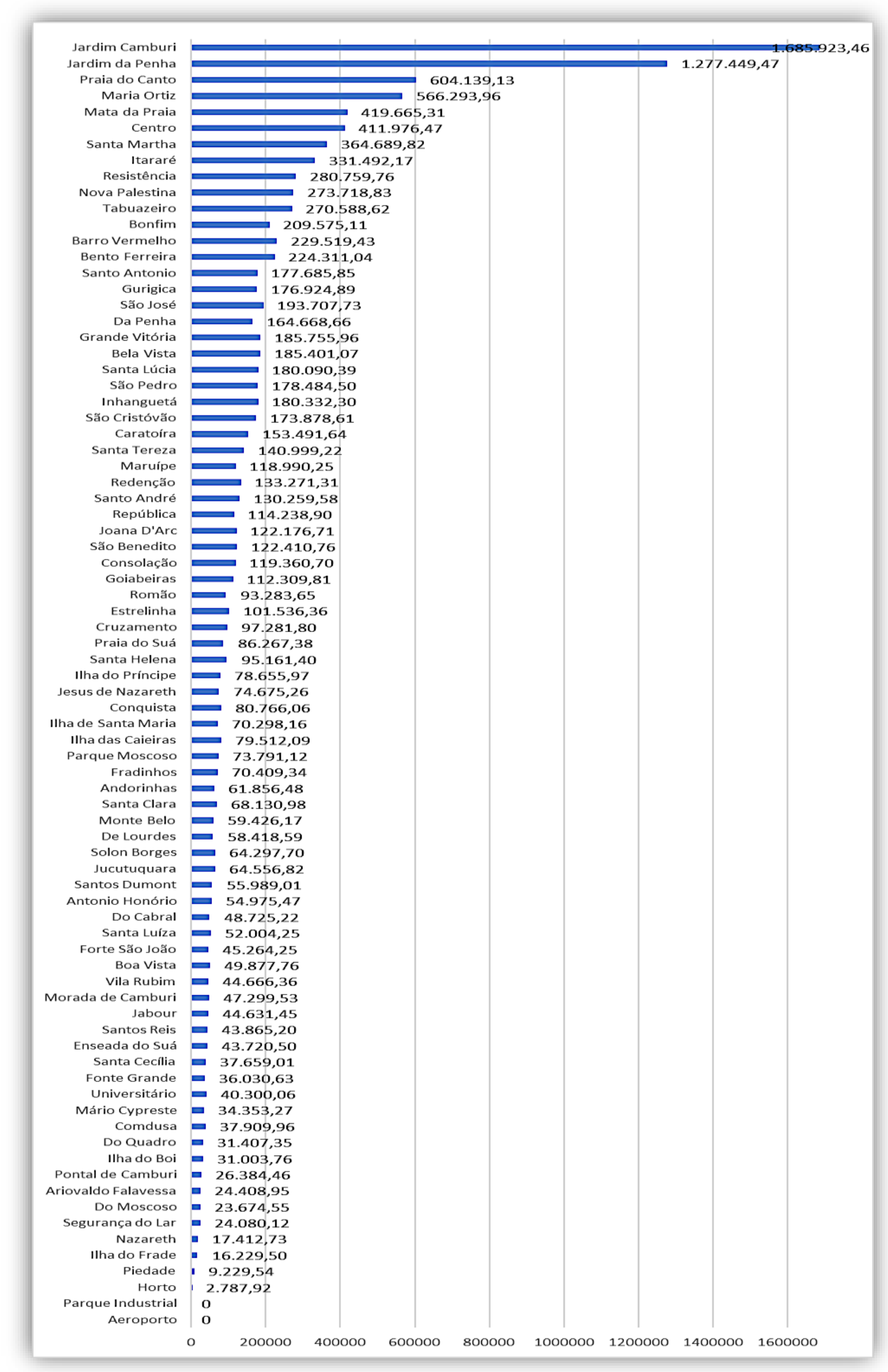


Citação (APA): Bittencourt, B. N. \& Servare Junior, M. W. J. (2021). Centro de gravidade para localizar centro de coleta para a economia circular do óleo de cozinha em Vitória, ES. Brazilian Journal of Production Engineering, 7(5), 194-206.

\section{RESULTADOS E ANÁLISES}

Para a implementação do método escolhido foram utilizadas as demandas de consumo que foram apresentadas nos Gráficos 1 e 2. Entretanto, a localização foi utilizada em longitude (X) e latitude (Y) facilitando assim a aplicação das fórmulas do método.

No desenvolvimento dos cálculos deste artigo as expressões (1) e (2) foram adaptadas, visto que no caso atual os custos seriam proporcionais o que não influenciaria no objetivo principal do estudo, sendo então descartados no presente momento de aplicação do método. Desse modo, utilizando o software Excel (2019) realizaram-se os cálculos para os valores das demandas em 5 anos e para as demandas em 15 anos de acordo com as expressões (3) e (4) apresentadas.

$$
\begin{aligned}
& x=\frac{\sum}{\sum} \quad V i x i \\
& y=\frac{\sum}{\sum} \quad V i y i \\
& \hline \sum \quad V i
\end{aligned}
$$

Sendo:

$V i=$ Demanda

$x i=$ Coordenada $\mathrm{x}$ dos bairros

$y i=$ Coordenada y dos bairros

Obtendo assim, os pontos $\mathrm{X}=-20,28719398262$ e $\mathrm{Y}=-40.3088108549544$ para o cenário de 5 anos e os pontos $\mathrm{X}=-20.2867329811762$ e $\mathrm{Y}=-40.3084073014506$ para o cenário de 15 anos como ponto ótimo (Tabela 1$)$.

Tabela 1. Coordenadas propostas a partir do método do Centro de Gravidade. Coordenadas para 5 anos Coordenadas para 15 anos $\mathrm{X}=-20,28719398262$ $\mathrm{X}=-20.2867329811762$ $Y=-40.3088108549544$ $\mathrm{Y}=-40.3084073014506$

Nos dois cenários apresentados as coordenadas geográficas obtidas correspondem ao endereço no bairro Santa Martha, conforme a tabela 2. O bairro proposto está entre os dez bairros com as maiores demandas do município, ocupando a sétima posição da lista com a demanda de 113.426,83 litros de óleo em 5 anos e 364.689,82 litros de óleo em 15 anos. Veja o Gráfico 3.

Figura 3. Dez bairros com a maior demanda de consumo de óleo de cozinha.

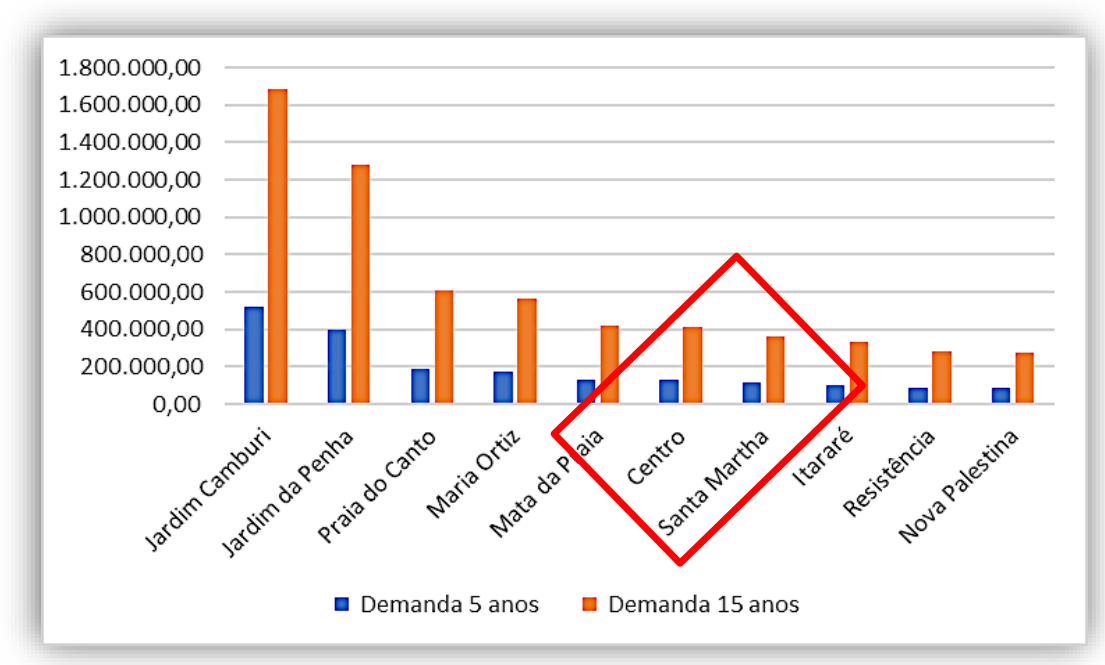


Citação (APA): Bittencourt, B. N. \& Servare Junior, M. W. J. (2021). Centro de gravidade para localizar centro de coleta para a economia circular do óleo de cozinha em Vitória, ES. Brazilian Journal of Production Engineering, 7(5), 194-206.

Além disso, o bairro Santa Martha encontra-se próximo a maioria dos 10 bairros selecionados com as maiores demandas, como pode ser identificado na Figura 4.

Figura 4. Localização dos 10 bairros com maior demanda em relação à localização proposta.

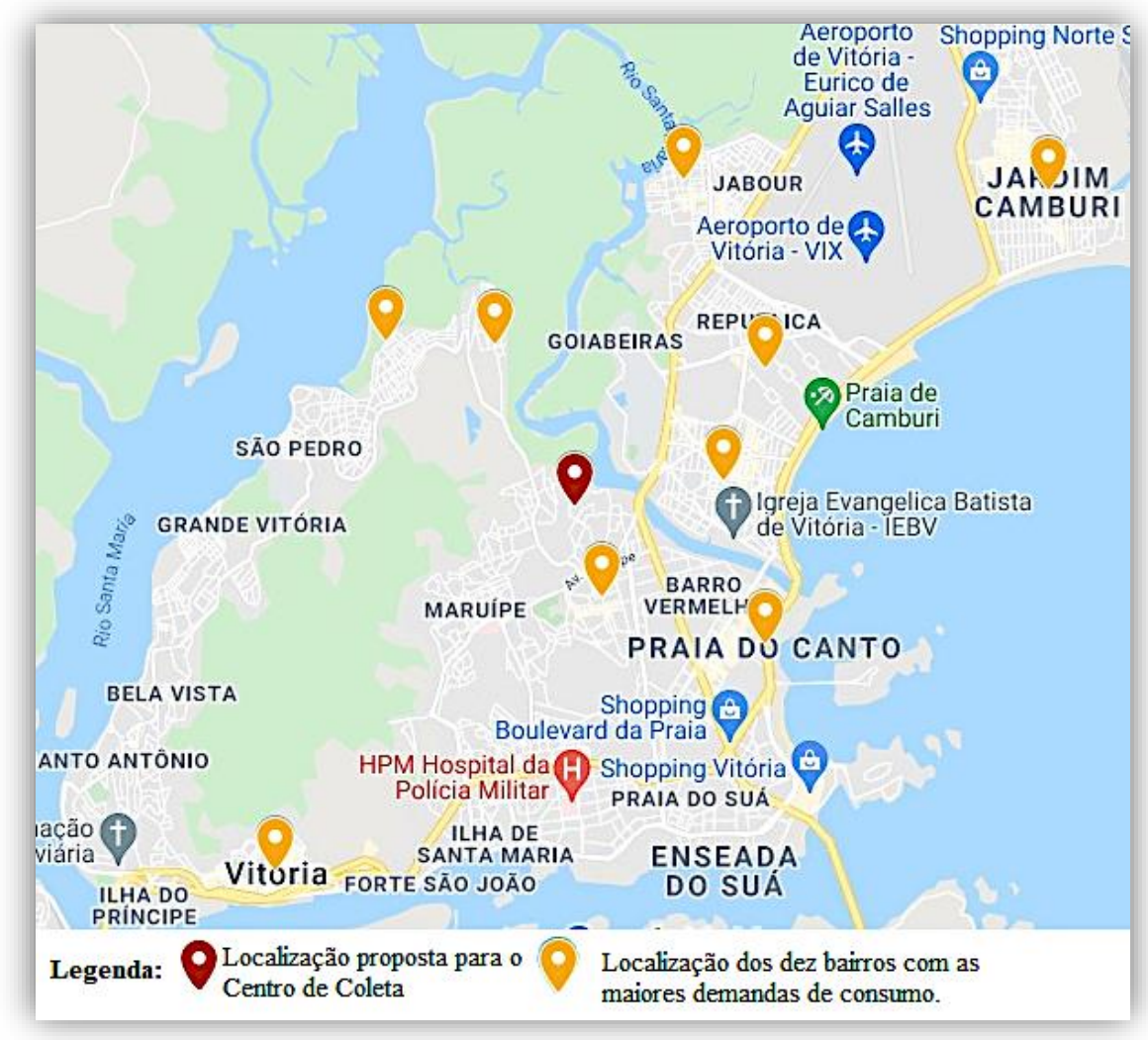

Fonte: Adaptado Google Maps (2021).

Em comparação a solução ótima proposta por Servare Junior e Cardoso (2020), a localização obtida através da utilização do método do Centro de Gravidade se deu próximo ao local indicado para o Centro de Triagem sugerido pelos autores. Em ambos os casos as localizações propostas respeitam os limites territoriais do município de Vitória.

\section{CONSIDERAÇÕES FINAIS}

Devido ao aumento da população e consequentemente o aumento do consumo, é necessária uma adaptação a novas práticas conscientes para tratar todos os resíduos gerados. Desse modo, são levantados cada vez mais temas relacionados ao meio ambiente, que ganham uma proporção maior à medida que a sociedade é despertada para a importância do descarte e da manipulação correta dos resíduos.

Através da revisão de literatura apresentada foi possível a identificação de trabalhos publicados que contribuem diretamente com o contexto da pesquisa. Sendo analisada a importância de um bom gerenciamento destes resíduos gerados, afirmando que a sociedade em geral ainda tem muito o que realizar sobre o tema. Reforçando a ideia de que é necessária a criação de mais práticas que atendam as demandas e mais centros de coletas destes resíduos.

Na busca de atingir o objetivo de indicar através do método do centro de gravidade a melhor localização de um ponto de coleta de óleo de cozinha, para a aplicação do método escolhido, 
Citação (APA): Bittencourt, B. N. \& Servare Junior, M. W. J. (2021). Centro de gravidade para localizar centro de coleta para a economia circular do óleo de cozinha em Vitória, ES. Brazilian Journal of Production Engineering, 7(5), 194-206.

foi necessário pesquisar as coordenadas geográficas dos bairros listados, bem como foram utilizadas as demandas dos litros de óleo de cozinha consumidos por cada bairro. Logo, através dos resultados obtidos com a implementação do método escolhido foi possível avaliar a localização do Centro de Coleta proposto.

Contudo, como limitação do presente trabalho destaca-se o método do Centro de Gravidade falha ao não analisar de forma mais criteriosa os aspectos qualitativos que também possuem grande impacto na decisão da localização. Para o desenvolvimento de trabalhos futuros, propõese a expansão da amostra para aplicação do método em outras regiões maiores, com o intuito de ampliar o volume de resíduos coletados, bem como ampliar a conscientização da sociedade em relação ao tema.

Além disso, sugere-se que sejam utilizados o método do Centro de Gravidade aplicado no presente trabalho combinado com o modelo aplicado por Servare Junior e Cardoso (2020) para o desenvolvimento de trabalhos futuros, com o intuito de propor uma solução mais ampla e detalhada.

\section{REFERÊNCIAS}

Abreu, A. B. G, Silva, L. A. O. de., Santos, M.V.M. dos., \& Santos, L. (2020). Análise da Gestão de Resíduos Sólidos Domésticos na Cidade de Macaé. Gestão de Riscos e Resiliência de cadeias de suprimentos em crises globais, Foz do Iguaçu, PR, Brasil, 40.

Almeida, C. D. S. (2014). Adequação do sistema de gerenciamento de resíduos sólidos de uma empresa cerâmica à política nacional conforme Lei 12305/2010. 1 v. TCC (Graduação) - Curso de Engenharia Ambiental, Universidade do Extremo Sul Catarinense, Criciúma.

Anais do Encontro Nacional de Engenharia de Produção - Enegep. Banco de Dados Online. Recuperado de http://www.abepro.org.br/publicacoes/

BRASIL, Lei $N^{\circ}$ 12.305, de 02 de agosto de 2010 - Política Nacional de Resíduos Sólidos (PNRS).

Brasil. Senado Federal. Projeto de Lei do Senado $n^{\circ} 75$, de 2017. Recuperado de http://www25.senado.leg.br/web/atividade/materias/-/materia/128531

Brazilian Journal of Engineering - BJPE. Banco de Dados Online. Recuperado de https://periodicos.ufes.br/bjpe

Fonseca, E. C. C. da, Barreiros, E. C. M., Melo, A. C. S., Carneiro, M. P., \& de Lucena Nunes, D. R. (2015). Evolução dos estudos de logística reversa realizados no contexto nacional: uma análise bibliométrica. Revista Produção Online, 15(4), 1457-1480.

Leitão, A. (2015). Economia circular: uma nova filosofia de gestão para o séc. XXI. Portuguese Journal of Finance, Management and Accounting, 1(2), 150-171.

Maia, A. M. G., Rodrigues, H. H. M., \& Moraes, J. R. S. F. (2020). Aplicação do método do centro de gravidade para localização de um centro de distribuição de uma fábrica de argamassa e rejunte. Economia Circular e suas interfaces com a Engenharia de Produção, Bauru, SP, Brasil, 27.

Martins, P. G., \& Laugeni, F. P. (2015) Administração da Produção. 3. ed. São Paulo: Saraiva 
Citação (APA): Bittencourt, B. N. \& Servare Junior, M. W. J. (2021). Centro de gravidade para localizar centro de coleta para a economia circular do óleo de cozinha em Vitória, ES. Brazilian Journal of Production Engineering, 7(5), 194-206.

Monteiro, C., Karpinski, J. A., Kuhl, M. R., \& Morozini, J. F. (2016). A gestão municipal de resíduos sólidos e as ações de sustentabilidade: um estudo realizado em um município do centro oeste do Paraná. Urbe. Revista Brasileira de Gestão Urbana, 9, 139-154.

Nascimento, V. F., Sobral, A. C., Andrade, P. R. D., \& Ometto, J. P. H. B. (2015). Evolução e desafios no gerenciamento dos resíduos sólidos urbanos no Brasil. Revista Ambiente \& Água, 10, 889-902.

Oliveira, B. F. de., Neto, J. O., kwaitkowski da Silva, J., \& Santoro, P. P. C. (2015) Uso Do Centro De Gravidade Para Localizar A Base Operacional Da Tpg Do Brasil. Otimização de recursos e desenvolvimento. Resende, RJ, Brasil,12.

Pitta Junior, O., Neto, M. N., Sacomano, J. B., \& Lima, J. L. A. (2009). Reciclagem do óleo de cozinha usado: uma contribuição para aumentar a produtividade do processo. In Internacional Workshop Advances In Cleaner Production, 2, 1-10.

Slack, N. et al. (2015) Administração da produção, 4ª Ed, São Paulo: Atlas.

Soares, I. R. C., Biagio, M. G., Gonçalves, M. F. S., \& Servare Junior, M. W. J. (2020). Localização de centro de triagem de papel para escolas do bairro da vila mariana, são paulo: location of paper sorting center for schools in vila mariana neighborhood, São Paulo. Brazilian Journal of Production Engineering, BJPE, 110-119.

Servare Junior, M. W. J., \& Cardoso, P. A. (2020). Modelo matemático para postergação de tempo no projeto de rede logística reversa com níveis de capacidade: mathematical modelling for time postponement in reverse network logistic design with capacity levels. Brazilian Journal of Production Engineering, BJPE, 01-22. https://doi.org/10.47456/bjpe.v6i7.32475 